\title{
Crecimiento y rendimiento del pepino holandés en ambiente protegido y con sustratos orgánicos alternativos ${ }^{1}$
}

\section{Growth and yield of Dutch cucumber grown in a protected environment and with alternative organic substrates}

\author{
Cinthya Meneses-Fernández², Gustavo Quesada-Roldán ${ }^{3}$
}

\begin{abstract}
Resumen
La sustitución de sustratos importados por materiales locales es una tendencia en varios sistemas de producción tecnificada en ambientes protegidos, dado que se reducen los costos y se valorizan subproductos agrícolas anteriormente considerados como desecho. El objetivo de este trabajo fue validar los efectos de diferentes sustratos en el crecimiento y la productividad de plantas de pepino holandés establecidas en invernadero. El trabajo se llevó a cabo entre octubre 2012 y enero 2013, en la Estación Experimental Fabio Baudrit, Alajuela, Costa Rica. Se empleó la variedad Fuerte y cuatro sustratos en mezcla (base volumen), elaborados a partir de materias primas locales como fibra de coco (FC), fibra de palma (FP), abono orgánico (ABO) y aserrín (AS), y un tratamiento comercial (testigo), constituido por tabletas de fibra de coco. Se evaluó el contenido de humedad en los sustratos, el crecimiento de la planta (fase vegetativa) y el rendimiento, según categorías de clasificación comercial por tamaño (S, M, L, XL y rechazo). El crecimiento de plantas de pepino holandés variedad Fuerte, fue mayor en sustratos con fibra de coco al $40 \%$ + fibra de palma al $40 \%$ + abono orgánico comercial al $20 \%$, y fibra de coco al $70 \%$ + abono orgánico comercial al $30 \%$, mientras que, la productividad de esos mismos dos sustratos fue de 15,57 y $15,44 \mathrm{~kg} / \mathrm{m}^{2}$, respectivamente. Ambos tratamientos fueron estadísticamente iguales al sustrato comercial de fibra de coco empacado en tabletas, que obtuvo un rendimiento de $14,77 \mathrm{~kg} / \mathrm{m}^{2}$. Resultado atribuido al aporte nutricional del abono orgánico alto en $\mathrm{K}$, Ca y Mg y a los efectos de la aireación y retención de humedad de las fibras.
\end{abstract}

Palabras clave: cultivo sin tierra, desechos agrícolas, Cucumis sativus, hortalizas cucurbitáceas, cultivo protegido.

\begin{abstract}
The replacement of imported substrates by local materials is a trend in several technified production systems, given the cost reduction and the importance of agricultural by-products, formerly considered to be waste. The main objective of this work was to validate the effect of different substrates on the growth and productivity of Dutch cucumber plants under greenhouse conditions. The study took place from October 2012 to January 2013, at the Agricultural Experiment Station Fabio Baudrit, Alajuela, Costa Rica. Variety Fuerte was used and four mixed substrates (volume ratio) made of local raw materials as coconut fiber (FC), oil palm leaf fiber (FP), organic compost
\end{abstract}

1 Recibido: 3 de mayo, 2017. Aceptado: 28 de junio, 2017. Este trabajo formó parte de la tesis de licenciatura de la primera autora. Universidad de Costa Rica, Facultad de Ciencias Agroalimentarias, Escuela de Agronomía, San José, Costa Rica.

2 Universidad de Costa Rica, Facultad de Ciencias Agroalimentarias, Escuela de Agronomía. cinmefdez@gmail.com

3 Universidad de Costa Rica, Estación Experimental Fabio Baudrit, Programa de Hortalizas. Alajuela, Costa Rica. gustavo.quesada@ucr.ac.cr 
(ABO), and sawdust (AS) as well as a commercial treatment (control) constituted by coco coir slabs were analyzed. Substrate water content, plant growth (vegetative phase), and yield were evaluated according to commercial size (S, $\mathrm{M}, \mathrm{L}, \mathrm{XL}$, and rejected). The growth of Dutch cucumber plants, variety Fuerte, was higher on substrates with coconut fiber $40 \%$ + oil palm leaf fiber $40 \%$ + organic compost $20 \%$, and coconut fiber $70 \%$ + organic compost $30 \%$, while yield of the same two substrates was 15.57 and $15.44 \mathrm{~kg} / \mathrm{m}^{2}$, respectively. Both treatments were statistically equal to the commercial coco coir substrate slabs with a yield of $14.77 \mathrm{~kg} / \mathrm{m}^{2}$. Result attributed to the nutritional contribution of the organic compost (high $\mathrm{K}, \mathrm{Ca}$, and $\mathrm{Mg}$ ) along with and to the effects of aeration and water retention of fibers.

Keywords: soilless culture, agricultural wastes, Cucumis sativus, cucurbit vegetables, protected cultivation.

\section{Introducción}

El cultivo en sustratos o cultivo sin suelo, ha cumplido un papel determinante en la mejora del rendimiento y la calidad de la producción hortícola, principalmente cuando se realiza en ambiente protegido. Debido a las propiedades fisicoquímicas de la turba de Sphagnum, esta se tornó el principal componente de muchos de los sustratos, es usada como padrón de comparación de los nuevos materiales alternativos que van surgiendo. Sin embargo, el costo de la turba es alto y se cuestiona el perjuicio ambiental derivado de su extracción al ser un recurso natural no renovable (Fermino, 2014). Como la demanda por sustratos y la diversificación de cultivos producidos bajo ambiente protegido está en crecimiento continuo, es necesario buscar otras opciones de materiales que cumplan con un mínimo de calidad sin dejar de lado las consideraciones ambientales y económicas (HernándezApaolaza et al., 2005; Acosta, 2012).

Una alternativa de sustratos es el aprovechamiento a partir de desechos agrícolas como el mesocarpio del coco, las hojas de la palma aceitera y diversos tipos de compostajes. Con esos materiales se puede agregar valor a productos que, en otra circunstancia, serían simplemente desechos. Tienen además la ventaja de ser, la mayoría de las veces, productos de bajo costo. La fibra de coco es el material más estudiado y utilizado con frecuencia como sustituto del musgo Sphagnum o musgo de turbera (peat moss), debido a sus similitudes (Abad et al., 2002; Hernández-Apaolaza et al., 2005). Esta fibra se caracteriza por la capacidad de retener de tres a cuatro veces su propio peso en agua, presenta alta porosidad, es leve, estable y con valores de $\mathrm{pH}$ y conductividad eléctrica dentro de los parámetros indicados para la mayoría de cultivos (Nelson, 2003; Takane et al., 2013).

La fibra resultante de las hojas de la palma aceitera (Elaeis guineensis Jacq) es un residuo orgánico de interés por ser un producto de desecho de fácil disponibilidad en Costa Rica. En los últimos años, se han realizado investigaciones para su posible uso en mezclas de sustratos (Méndez, 2007; Meneses-Fernández y QuesadaRoldán, 2015). Es un medio que puede contribuir con la aireación de un sustrato, pues posee partículas grandes de más de $2 \mathrm{~mm}$ y una porosidad mayor al 90\%. Sin embargo, a su vez tiene una capacidad de retención de agua limitada de alrededor del 30\% (Méndez, 2007).

Los diversos tipos de compostajes también son fuente común de sustratos. Estos materiales cuando son sometidos a procesos de aireación, temperatura, humedad y pasado el tiempo para completar su maduración, contribuyen con nutrientes y aumentan el contenido de agua disponible en mezcla de sustratos; su capacidad de intercambio catiónico también es alta (Handreck y Black, 2002). Sin embargo, debe tenerse cuidado al usarlos, pues si no se han producido adecuadamente se corre el riesgo de que contengan patógenos o favorezca el propágulo de

malezas. Un compost que no esté totalmente terminado presenta también valores elevados de tasa de reducción de nitrógeno, concentración de amonio, $\mathrm{pH}$ y conductividad eléctrica (CE), además de elevar la temperatura del sustrato (Alvarado y Solano, 2002; Handreck y Black, 2002). 
La idea de revalorizar materiales considerados como subproductos agrícolas, ha demostrado ser efectiva en la producción en sustratos y ha favorecido una demanda creciente por potencial materia prima que anteriormente llegaba a considerarse como desecho (Quintero et al., 2011). Sin embargo, al momento de realizar una mezcla de materiales para conformar un nuevo sustrato, debe considerarse la interacción de las materias primas que resulta en la generación de un medio con propiedades físicas y químicas diferentes a las de sus componentes originales. Esta propiedad de la no aditividad de los sustratos descrita por Fonteno (1996), es la que hace necesario estudiar las características fisicoquímicas que resultan de una mezcla elaborada y la consecuente respuesta en el crecimiento y rendimiento de las plantas que se establecen en esos medios de crecimiento.

La capacidad de retener humedad de los sustratos es uno de los criterios de selección cuando se evalúan diversos materiales para componer una mezcla que pueda ser usada como sustrato, dado que esa retención define en gran medida la disponibilidad de agua para los cultivos (Acosta, 2012). Un adecuado suministro de agua define en buena medida el crecimiento, desarrollo y producción de las plantas, al ser el agua el vehículo que permite la incorporación de los nutrimentos presentes en la solución que será absorbida por las raíces de la planta (Handreck y Black, 2002).

El uso de estructuras de ambientes protegidos en la producción de hortalizas como pepino, ofrece un potencial valioso para los productores, pues existen mercados internacionales en crecimiento que tienen interés por este producto, y que son a la vez exigentes en aspectos sanitarios y de calidad (Quirós, 2015). En los últimos años, se ha incrementado la importancia del pepino como una opción altamente rentable, al ser un cultivo de ciclo corto, muy productivo y de relativo fácil manejo. En México, este cultivo se encuentra entre los cinco de mayor importancia en ambientes protegidos (Hernández-González et al., 2014), mientras que en Estados Unidos es el segundo (Shaw et al., 2007); en Costa Rica se ha intentado impulsar la producción del pepino holandés para exportación, específicamente las variedades Roxinante y Fuerte, principalmente por la posibilidad de acceder al mercado estadounidense y canadiense, aprovechando las ventanas de mercado (Mora et al., 2007). Mejoras en la tecnificación de los procesos productivos en este cultivo son requeridos, y definir un sustrato local ayudaría en ese mejoramiento y contribuiría a reducir la dependencia por sustratos importados. El objetivo de este trabajo fue validar los efectos de diferentes sustratos en el crecimiento y productividad de plantas de pepino holandés establecidas en condiciones de invernadero.

\section{Materiales y métodos}

La validación de sustratos se hizo en el invernadero multicapilla de la Estación Experimental Agrícola Fabio Baudrit Moreno (EEAFBM), de la Universidad de Costa Rica, ubicado en el Barrio San José de Alajuela, a 840 msnm, y a $10^{\circ} 00^{\prime} 396^{\prime}$ ' de latitud norte y $84^{\circ} 15^{\prime} 970^{\prime}$ de longitud oeste. El invernadero es semiclimatizado, con cuatro módulos de 9,6 $\mathrm{m}$ de ancho, $4 \mathrm{~m}$ de altura a la canoa y abertura cenital automatizada para regular la temperatura interna. El experimento se llevó a cabo entre octubre de 2012 y enero de 2013.

Se evaluaron cuatro sustratos en mezcla y un sustrato comercial de tabletas de fibra de coco de un metro de largo por $18 \mathrm{~cm}$ de alto, usado como tratamiento testigo (Tableta FC). Las cuatro mezclas usadas fueron las siguientes: fibra de coco con fibra de palma y abono orgánico comercial, este abono elaborado a base de bagazo de caña de azúcar y broza de café (FC 40\% + FP 40\% + ABO 20\%); fibra de coco con abono orgánico comercial (FC $70 \%$ + ABO 30\%); fibra de coco con fibra de palma (FC 50\% + FP 50\%); fibra de palma con aserrín de melina (FP $70 \%+$ AS 30\%). El porcentaje indicado entre paréntesis en cada tratamiento, se refiere al volumen proporcional en base $\mathrm{v} / \mathrm{v}$ en el que determinado componente se encontraba en la mezcla. Las cuatro mezclas se seleccionaron previamente, por presentar características fisicoquímicas de potencial interés para uso como sustrato hortícola (las propiedades de los sustratos pueden consultarse en Meneses-Fernández y Quesada-Roldán (2015)). 
Se utilizaron plantas de pepino tipo holandés (Cucumis sativus L.) variedad Fuerte, debido a experiencias previas positivas del mismo genotipo en ciclos de producción en invernaderos del cantón de Zarcero, Alajuela, Costa Rica (Mora et al., 2007). El material es partenocárpico y no requiere de agentes polinizadores para la formación de frutos. El almácigo se hizo en las propias instalaciones de la EEFBM, con musgo Sphagnum o musgo de turbera (peat moss), como medio de crecimiento. A partir del momento que empezó el desarrollo de la segunda hoja verdadera (aproximadamente doce días después de la siembra), las plántulas se trasplantaron en contenedores plásticos de 7 1, excepto el tratamiento testigo que se trasplantó en la tableta comercial de fibra de coco.

Se trabajó en una área de $252 \mathrm{~m}^{2}$ aproximadamente, conformada por doce hileras de $14 \mathrm{~m}$ de longitud cada una. Las hileras de los extremos, así como las dos primeras y dos últimas plantas de cada hilera, constituyeron el área de borde del experimento. Cada hilera estaba conformada por los cinco tratamientos y diez plantas por tratamiento. Cada unidad experimental contempló dos hileras, por tanto, veinte plantas por unidad experimental por tratamiento, para un total de cien plantas por tratamiento. Para garantizar condiciones representativas, se implementó como diseño experimental un cuadrado latino con cinco repeticiones $(5 \times 5)$ y se aleatorizaron los cinco tratamientos. La distancia de siembra entre plantas fue de $0,25 \mathrm{~m}$ y entre hileras de 1,6 m, para una densidad de 2,5 plantas $/ \mathrm{m}^{2}$.

La temperatura y humedad relativa dentro del invernadero se obtuvieron mediante sensores digitales automatizados e integrados que, registraron las condiciones climáticas diarias promedio a cada hora. El promedio de temperatura y humedad relativa del ciclo entero fue respectivamente de $23,8{ }^{\circ} \mathrm{C}$ (máx. $38,6-\min .15,5^{\circ} \mathrm{C}$ ) y 72,6 \% (máx. 98,5 - min. 34,8\%).

Para el manejo agronómico del cultivo se siguieron estrategias de manejo integrado de plagas (MIP) para el control de patógenos (principalmente mildiú polvoso) y plagas (con destaque para mosca blanca y ácaros), y deshijas semanales como poda de formación para mantener una única guía principal de crecimiento. El riego y la nutrición de las plantas se hizo a través de programas de fertirriego diario (Cuadro 1), mediante sistemas presurizados de riego por goteo, fraccionando la dosificación en tres fases fenológicas distintas: desarrollo vegetativo, floración y producción de frutos. En promedio a lo largo del experimento, se suministró 1,2 1 de solución nutritiva. Todas las anteriores variables se manejaron por igual para todos los tratamientos.

Cuadro 1. Programa de fertirriego (macroelementos en mg/l) empleado para la producción de pepino holandés variedad Fuerte, en condiciones de invernadero. Estación Experimental Agrícola Fabio Baudrit Moreno, Alajuela, Costa Rica. 2013.

Table 1. Fertigation program (macronutrients on $\mathrm{mg} / \mathrm{l}$ ) used for the production of Dutch cucumber, variety Fuerte, under greenhouse conditions. Fabio Baudrit Moreno Agricultural Experiment Station, Alajuela, Costa Rica. 2013.

\begin{tabular}{cccc}
\hline & \multicolumn{3}{c}{ Etapa fenológica } \\
\cline { 2 - 4 } Nutrimento & Fase vegetativa & Floración & Fructificación \\
\cline { 2 - 4 } & $\mathbf{1 - 2 0}$ DDT & $\mathbf{2 1 - 3 3 ~ D D T}$ & $\mathbf{3 4 - 9 2}$ DDT \\
\hline Nitrógeno & 150 & 160 & 175 \\
Fósforo & 55 & 50 & 50 \\
Potasio & 220 & 260 & 290 \\
Calcio & 160 & 165 & 175 \\
Magnesio & 40 & 50 & 55 \\
pH: & 6,2 & 6,3 & 6,3 \\
C.E: & 1,5 & 1,8 & 2,0 \\
\hline
\end{tabular}

Para comprobar la retención de agua de los sustratos y la capacidad que poseen de hacer asequible la solución con el agua y nutrientes requeridos por las plantas, se determinó el contenido de humedad (\% de agua) en tiempo 
real de los sustratos, con el empleo de un sensor de tecnología TDR (Time Domain Reflectometry), que se basa en la determinación de humedad del medio, mediante impulsos eléctricos. Las mediciones se realizaron a los 23 y a los 53 días después del trasplante, y se determinaron en dos horarios del día, a las 8:00 am y a las 12:00 md. Las lecturas se tomaron en momentos anteriores a la irrigación, para evitar la influencia de eventos de riego cercanos a las mediciones.

Las evaluaciones de crecimiento se hicieron a los 10, 19 y 25 días después del trasplante, dado que la fase vegetativa en cultivos anuales como el pepino corresponde al periodo de mayor crecimiento de la planta (Larcher, 2000). Se evaluó la altura de la planta (en cm desde la base hasta el meristema apical), el número y tamaño de las hojas completamente desarrolladas (medido según el largo en cm desde la inserción del peciolo en la lámina foliar hasta el ápice de la hoja) y la cantidad de flores y frutos. Además, se realizó un análisis foliar a los cuarenta días después del trasplante (Cuadro 2), que mostró que las plantas no presentaban problemas nutricionales estando los valores dentro del rango óptimo para pepino. Eso se hizo para comprobar el adecuado suministro de nutrientes y que la respuesta de la planta de pepino se debiere a los sustratos y no a un posible efecto de la solución nutritiva. Se tomó como muestra la hoja número cinco (desde el meristema hasta la base de la planta) en plantas al azar de cada unidad experimental y se llevaron para análisis al Laboratorio de Suelos y Foliares del Centro de Investigaciones Agronómicas de la Universidad de Costa Rica.

Cuadro 2. Porcentaje de macronutrimentos y concentración de micronutrimentos $(\mathrm{mg} / \mathrm{kg})$ a los cuarenta días después del trasplante, en hojas de plantas de pepino holandés variedad Fuerte, establecidas en diferentes sustratos, en condiciones de invernadero. Estación Experimental Agrícola Fabio Baudrit Moreno, Alajuela, Costa Rica. 2013.

Table 2. Percentage of macronutrients and concentration of micronutrients ( $\mathrm{mg} / \mathrm{kg}), 40$ days after transplant, in leaves of Dutch cucumber plants, variety Fuerte, grown on different substrates under greenhouse conditions. Fabio Baudrit Moreno Agricultural Experiment Station, Alajuela, Costa Rica. 2013.

\begin{tabular}{|c|c|c|c|c|c|c|c|c|c|c|c|}
\hline \multirow[b]{2}{*}{ Sustrato } & \multicolumn{6}{|c|}{$\%$} & \multicolumn{5}{|c|}{$\mathrm{mg} / \mathrm{kg}$} \\
\hline & $\mathbf{N}$ & $\mathbf{P}$ & $\mathbf{C a}$ & Mg & $\mathbf{K}$ & $\mathbf{S}$ & $\mathbf{F e}$ & $\mathbf{C u}$ & Zn & Mn & B \\
\hline $\mathrm{FC} 40 \%+\mathrm{FP} 40 \%+\mathrm{ABO} 20 \%$ & 6,51 & 0,73 & 0,92 & 0,40 & 4,06 & 0,58 & 91 & 7 & 53 & 74 & 29 \\
\hline FC $50 \%+$ FP $50 \%$ & 6,29 & 0,86 & 0,70 & 0,36 & 5,02 & 0,62 & 83 & 5 & 47 & 43 & 29 \\
\hline $\mathrm{FC} 70 \%+\mathrm{ABO} 30 \%$ & 6,39 & 0,76 & 0,89 & 0,41 & 3,98 & 0,62 & 97 & 7 & 39 & 64 & 31 \\
\hline $\mathrm{FP} 70 \%+\mathrm{AS} 30 \%$ & 6,08 & 0,74 & 0,72 & 0,36 & 3,98 & 0,64 & 86 & 6 & 45 & 45 & 29 \\
\hline Tableta FC & 5,92 & 0,89 & 0,56 & 0,41 & 4,79 & 0,57 & 78 & 5 & 47 & 50 & 29 \\
\hline
\end{tabular}

FC $40 \%$ + FP 40\% + ABO 20\%= fibra de coco al 40\% + fibra de palma al 40\% + abono orgánico comercial al 20\% / coconut fiber $40 \%$ + oil palm leaf fiber $40 \%+$ commercial organic compost $20 \%$.

FC 50\% + FP 50\% = fibra de coco al 50\% + fibra de palma al 50\% / coconut fiber 50\% + oil palm leaf fiber 50\%.

FP $70 \%+\mathrm{ABO} 30 \%=$ fibra de palma al 70\% + abono orgánico comercial al 30\% / oil palm leaf 70\% + organic compost 30\%.

FP 70\% + AS 30\%= fibra de palma al 70\% + aserrín al 30\% / oil palm leaf 70\% + sawdust 30\%.

Tableta FC= tableta comercial de fibra de coco / commercial coconut coir slabs.

El fruto de pepino holandés producido, se caracterizó por la ausencia de semillas, tamaño alargado y cáscara delgada, lisa y brillante. La productividad se evaluó a partir de la clasificación de los frutos para exportación en categorías comerciales: S, M, L, XL y rechazo (Quesada et al., 2008). Los tamaños de cada categoría, considerando largo de la fruta y criterios de tamaño definidos por el mercado, fueron: $\mathrm{S}=28,5$ a 30,5 cm (mínimo aceptado por los mercados internacionales para exportación); $\mathrm{M}=30,6$ a $33 \mathrm{~cm} ; \mathrm{L}=33,1$ a $35,5 \mathrm{~cm} ; \mathrm{XL}=$ más de $35,5 \mathrm{~cm}$. Frutos en la categoría rechazo fueron aquellos con deformaciones, curvaturas o problemas en el cierre apical o fitosanitarios, aspectos que ocasionan la pérdida de su valor comercial. 
El tratamiento estadístico de la información, se realizó a partir de un análisis de varianza y pruebas de separación de medias por LSD Fisher, empleando como paquete estadístico el programa InfoStat (InfoStat Profesional v.1.1. Facultad de Ciencias Agropecuarias, Universidad Nacional de Córdoba, Argentina).

\section{Resultados}

\section{Contenido de humedad del sustrato}

Tanto en los días evaluados como en los horarios definidos, se encontraron diferencias significativas debido al contenido de humedad del sustrato (Cuadro 3).

Cuadro 3. Porcentaje de humedad (\%) en sustratos con plantas de pepino holandés variedad Fuerte en invernadero, a los 23 y 53 días después del trasplante (DDT), en dos horarios del día. Estación Experimental Agrícola Fabio Baudrit Moreno. Alajuela, Costa Rica. 2013.

Table 3. Percentage of water retention (\%) on substrates with Dutch cucumber plants, variety Fuerte, under greenhouse conditions, 23 and 53 days after transplant at two times of the day. Fabio Baudrit Moreno Agricultural Experiment Station. Alajuela, Costa Rica. 2013.

\begin{tabular}{|c|c|c|c|c|}
\hline \multirow[b]{3}{*}{ Tratamiento } & \multicolumn{4}{|c|}{ Periodo de medición } \\
\hline & \multicolumn{2}{|c|}{23 DDT } & \multicolumn{2}{|c|}{53 DDT } \\
\hline & 8:00 am & $12: 00 \mathrm{md}$ & 8:00 am & $12: 00 \mathrm{md}$ \\
\hline $\mathrm{FC} 40 \%+\mathrm{FP} 40 \%+\mathrm{ABO} 20 \%$ & $27,47 \mathrm{~b}^{1}$ & $27,61 \mathrm{~b}$ & $26,15 \mathrm{a}$ & $24,26 \mathrm{~b}$ \\
\hline FC $50 \%+$ FP $50 \%$ & $21,05 \mathrm{c}$ & $19,32 \mathrm{c}$ & $21,4 \mathrm{~b}$ & $20,42 \mathrm{c}$ \\
\hline $\mathrm{FC} 70 \%+\mathrm{ABO} 30 \%$ & $31,05 \mathrm{a}$ & 31,14 a & 28,56 a & 29,49 a \\
\hline $\mathrm{FP} 70 \%+\mathrm{AS} 30 \%$ & $8,57 \mathrm{~d}$ & $8,91 \mathrm{~d}$ & $6,56 \mathrm{c}$ & $8,9 \mathrm{~d}$ \\
\hline Tableta FC & $32,22 \mathrm{a}$ & $29,15 \mathrm{a}$ & 25,89 a & $27,04 \mathrm{a}$ \\
\hline
\end{tabular}

${ }^{1}$ Medias con igual letra en la misma columna, no difieren estadísticamente según prueba de separación de rangos LSD Fisher ( $\mathrm{p} \leq 0,05$ ). / Means with the same letter in the same column do not differ statistically according to Fishers least significant difference test LSD $(\mathrm{p} \leq 0.05)$.

FC $40 \%$ + FP 40\% + ABO 20\% = fibra de coco al 40\% + fibra de palma al 40\% + abono orgánico comercial al 20\% / coconut fiber $40 \%$ + oil palm leaf fiber $40 \%+$ commercial organic compost $20 \%$.

FC 50\% + FP 50\% = fibra de coco al 50\% + fibra de palma al 50\% / coconut fiber 50\% + oil palm leaf fiber 50\%.

FP $70 \%$ + ABO 30\%= fibra de palma al 70\% + abono orgánico comercial al 30\% / oil palm leaf fiber 70\% + commercial organic compost $30 \%$.

FP 70\% + AS 30\%= fibra de palma al 70\% + aserrín al 30\% / oil palm leaf fiber 70\% + sawdust 30\%.

Tableta FC= tableta comercial de fibra de coco / commercial coco coir slabs.

Los tratamientos Tableta FC (testigo comercial) y FC 70\% + ABO 30\%, ofrecieron consistentemente una mayor retención de humedad y se destacaron estadísticamente como los mejores sustratos para esta variable en todos los periodos evaluados, seguidos del tratamiento FC 40\% + FP 40\% + ABO 20\%, que incluso estuvo también entre los de mayor retención en la evaluación de las 8:00 AM a los 53 DDT. Lo anterior confirma la capacidad que tiene la fibra de coco para retener humedad.

El tratamiento de FP70\% + AS30\% fue el que tuvo significativamente el menor porcentaje de humedad en el sustrato, menos de $10 \%$ en todas las mediciones, diferenciándose de todos los otros tratamientos. Otro tratamiento 
donde se reflejó poca retención de humedad y también con significancia estadística fue el de FC50\% + FP50\%, siempre con valores cercanos al 20\%. La presencia de fibra de palma en al menos $50 \%$ de la mezcla resultó determinante para observar poca retención de humedad en los sustratos.

\section{Crecimiento}

La evolución en el crecimiento de las plantas de pepino en el primer mes de establecido, según las variables de altura de planta y tamaño y número de hojas, se presenta en la Figura 1. A los 10 DDT hubo diferencias estadísticas en el desarrollo de las plantas, debido al tipo de sustrato usado, pero es a partir de los 19 DDT que estas diferencias fueron más marcadas. Los sustratos FC40\% + FP40\% + ABO20\% y FC70\% + ABO30\%, con un promedio de 105 y $110 \mathrm{~cm}$ de altura a los 25 días después de trasplante, fueron los de mejor desempeño en las tres variables citadas, similares estadísticamente entre sí, pero distintos significativamente de los otros tres tratamientos.

El sustrato donde fue registrado el menor crecimiento de las plantas en las fechas evaluadas, fue FP70\% + AS30\%. El sustrato FC50\% + FP50\% y el tratamiento testigo (tabletas comerciales de fibra de coco) tuvieron resultados intermedios; es decir, fueron similares entre sí, pero inferiores estadísticamente a los mejores sustratos evaluados y superiores cuando fueron comparados contra el tratamiento donde se registró menor crecimiento.

El inicio de floración se registró a partir de los 19 DDT en los tratamientos que tenían abono orgánico en la mezcla como el FC 40\% + FP 40\% + 20\% ABO, con un promedio de dos flores por planta, y el FC $70 \%+\mathrm{ABO}$ $30 \%$, con 1,2 flores por planta, mismos sustratos donde se presentó mayor crecimiento vegetativo (Cuadro 4). El tratamiento testigo (tableta de fibra de coco), también resultó similar estadísticamente que el anterior. Sustratos con predominancia de fibra de palma, no reportaron ninguna flor a ese momento. Para la evaluación a los 25 DDT, la mayoría de tratamientos se igualaron, con un promedio de alrededor de tres flores por planta, excepto para FP 70\% + AS 30\% que fue el único estadísticamente distinto a los demás con solo 1,7 flores por planta.

Respecto al número de frutos formados a los 25 DDT, los sustratos FC 40\% + FP 40\% + 20\% ABO y FC $70 \%$ $+\mathrm{ABO} 30 \%$, fueron los mejores, ya que cada planta tuvo en promedio alrededor de tres frutos, mostrando alta precocidad, lo que concuerda con los resultados anteriores de crecimiento y desarrollo del cultivo en esos sustratos. El tratamiento FP 70\% + AS 30\% fue el único que no presentó frutos a ese momento, pero aun así no se diferenció estadísticamente de los tratamientos de FC 50\% + FP 50\% y tableta comercial (testigo).

\section{Producción}

El periodo de cosecha se extendió de los 42 hasta los 99 DDT y se caracterizó por una gran producción de frutos tamaño S, principalmente en las tres primeras semanas de cosecha. Frutos de tamaño L y XL, de mayor valor comercial, solo se observaron en un pico de producción a los 67 DDT y posteriormente, hacia el final del ciclo productivo, en las últimas tres semanas de cosecha, ya con la planta iniciando su proceso de senescencia. La anterior distribución en el tiempo de la cosecha por categorías (datos no presentados), fue muy similar para todos los tratamientos evaluados y no hubo significancia estadística (Cuadro 5).

Los tratamientos con mayor número de frutos categoría S fueron FC 40\% + FP 40\% + ABO 20\% y FC 70\% + $\mathrm{ABO} 30 \%$, similares estadísticamente entre sí, pero diferentes significativamente de los demás. En ambos tratamientos el porcentaje total de frutos categoría $\mathrm{S}$ fue cercano al 50\%. Los tratamientos de Tableta FC y FP $70 \%+$ AS 30\% tuvieron estadísticamente el menor número de frutos S, correspondiente a un porcentaje entre 35 y $38 \%$ del total de frutos. En el caso de los frutos categoría M, el comportamiento en número de frutos entre los tratamientos fue muy semejante, únicamente el sustrato FP 70\% + AS 30\% mostró estadísticamente menor número de frutos, con el $14 \%$ de los frutos totales. En la categoría tamaño L, la mayor produción se dio en los sustratos Tableta FC y FC 70\% + ABO 30\%, iguales entre sí, aunque FC 40\% + FP 40\% + ABO 20\% y FC 50\% + FP 50\% 


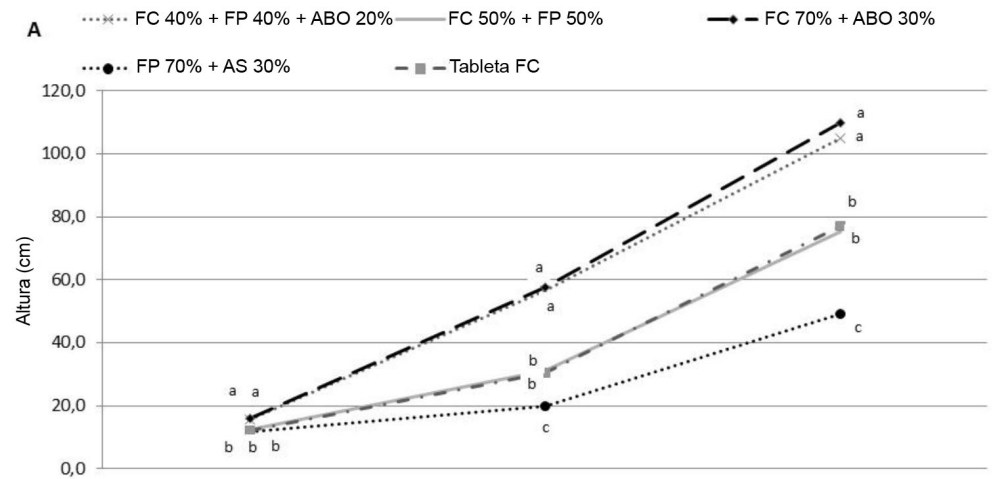

B

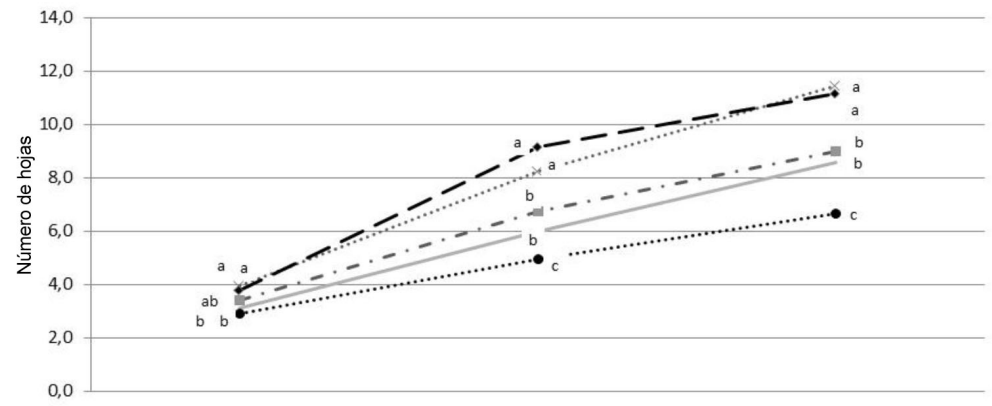

C

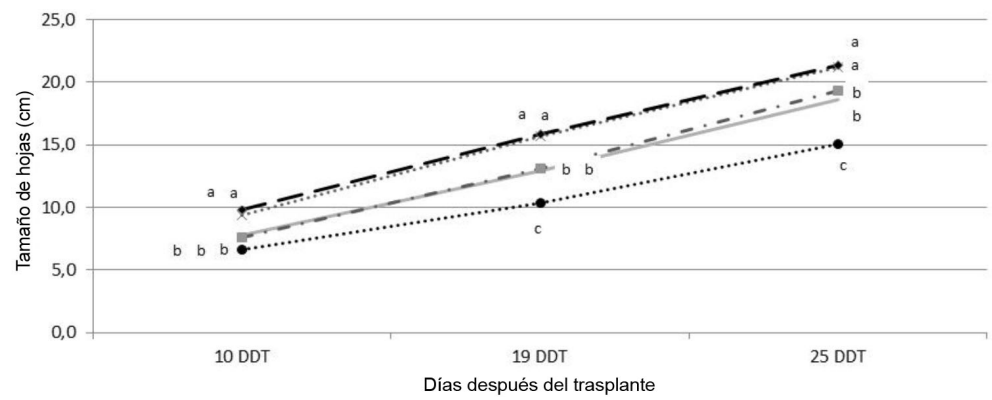

Figura 1. Altura (A), número de hojas (B) y tamaño de hojas (C) en plantas de pepino holandés variedad Fuerte, establecidas en diferentes sustratos en invernadero en tres momentos del ciclo de cultivo. Estación Experimental Agrícola Fabio Baudrit Moreno, Alajuela, Costa Rica. 2013.

Medias con igual letra no difieren estadísticamente entre si, según prueba de separación de rangos LSD Fisher $(\mathrm{p} \leq 0,05)$. FC $40 \%+\mathrm{FP} 40 \%$ + ABO 20\% = fibra de coco al $40 \%$ + fibra de palma al $40 \%$ + abono orgánico comercial al 20\%; FC $50 \%$ + FP 50\%= fibra de coco al 50\% + fibra de palma al 50\%; FP 70\% + ABO 30\% = fibra de palma al 70\% + abono orgánico comercial al 30\%; FP 70\% + AS 30\%= fibra de palma al 70\% + aserrín al 30\%; Tableta FC= tableta comercial de fibra de coco.

Figure 1. Height (A), number of leaves (B) and leaves size (C) in Dutch cucumber plants, variety Fuerte, grown on different substrates under greenhouse conditions in three crop stages. Fabio Baudrit Moreno Agricultural Experiment Station, Alajuela, Costa Rica. 2013.

Means with the same letter does not differ statistically according to Fishers least significant difference test $\operatorname{LSD}(\mathrm{p} \leq 0.05)$. $\mathrm{FC} 40 \%+\mathrm{FP} 40 \%+\mathrm{ABO} 20 \%=$ coconut fiber $40 \%$ + oil palm leaf fiber 40\% +commercial organic compost 20\%; FC 50\% + FP 50\%= coconut fiber 50\% + oil palm leaf fiber 50\%; FP 70\% + ABO 30\%= oil palm leaf fiber 70\% + commercial organic compost 30\%; FP 70\% + AS 30\%= oil palm leaf fiber 70\% + sawdust 30\%; Tableta FC $=$ commercial coco coir slabs. 
Cuadro 4. Número de flores y frutos al inicio de la fase reproductiva, en plantas de pepino holandés variedad Fuerte establecidas en diferentes sustratos. Estación Experimental Agrícola Fabio Baudrit Moreno. Alajuela, Costa Rica. 2013.

Table 4. Number of flowers and fruits at the beginning of the reproductive stage in Dutch cucumber plants, variety Fuerte, grown on different substrates. Fabio Baudrit Moreno Agricultural Experiment Station. Alajuela, Costa Rica. 2013.

\begin{tabular}{lcccccc}
\hline & & \multicolumn{4}{c}{ Tratamiento } \\
\cline { 2 - 7 } Variable & DDT & $\begin{array}{c}\text { FC 40\% + FP40\% } \\
\mathbf{+} \text { ABO 20\% }\end{array}$ & $\begin{array}{c}\text { FC 50\% + FP } \\
\mathbf{5 0 \%}\end{array}$ & $\begin{array}{c}\text { FC 70\% + ABO } \\
\mathbf{3 0 \%}\end{array}$ & $\begin{array}{c}\text { FP 70\% + AS } \\
\mathbf{3 0 \%}\end{array}$ & Tableta FC \\
\hline Número de flores & 19 & $2 \mathrm{a}^{1}$ & $0 \mathrm{~b}$ & $1,2 \mathrm{~b}$ & $0 \mathrm{~b}$ & $1 \mathrm{~b}$ \\
& 25 & $3,2 \mathrm{a}$ & $3,3 \mathrm{a}$ & $2,6 \mathrm{ab}$ & $1,7 \mathrm{~b}$ & $2,7 \mathrm{a}$ \\
\hline Número de frutos & 25 & $2,9 \mathrm{a}$ & $0,0 \mathrm{~b}$ & $3,4 \mathrm{a}$ & $0 \mathrm{~b}$ & $1 \mathrm{~b}$ \\
\hline
\end{tabular}

${ }^{1}$ Medias con igual letra en la misma línea, no difieren estadísticamente según prueba de separación de rangos LSD Fisher (P $\left.\leq 0,05\right)$. / Means with the same letter in the same column do not differstatistically according to Fishers least significant difference test LSD $(\mathrm{p} \leq 0,05)$.

FC $40 \%$ + FP 40\% + ABO 20\%= fibra de coco al 40\% + fibra de palma al 40\% + abono orgánico comercial al 20\% / coconut fiber $40 \%$ + oil palm leaf fiber $40 \%+$ commercial organic compost $20 \%$.

FC 50\% + FP 50\% = fibra de coco al 50\% + fibra de palma al 50\% / coconut fiber 50\% + oil palm leaf fiber 50\%.

$\mathrm{FP} 70 \%+\mathrm{ABO} 30 \%=$ fibra de palma al 70\% + abono orgánico comercial al 30\% / oil palm leaf fiber 70\% + commercial organic compost $30 \%$.

FP 70\% + AS 30\% = fibra de palma al 70\% + aserrín al 30\% / oil palm leaf fiber 70\% + sawdust 30\%.

Tableta FC= tableta comercial de fibra de coco / commercial cococoir slabs.

Cuadro 5. Número promedio de frutos por categoría comercial en plantas de pepino holandés variedad Fuerte, establecidas en diferentes sustratos en condiciones de invernadero. Estación Experimental Agrícola Fabio Baudrit Moreno, Alajuela, Costa Rica. 2013.

Table 5. Average number of fruits from commercial categories in Dutch cucumber plants, variety Fuerte, grown on different substrates under greenhouse conditions. Fabio Baudrit Moreno Agricultural Experiment Station, Alajuela, Costa Rica. 2013.

\begin{tabular}{lccccc}
\hline & \multicolumn{4}{c}{ Categoría Comercial } \\
\cline { 2 - 6 } Tratamiento & S & M & L & XL & Rechazo \\
\hline FC 40\% + FP 40\%+ABO 20\% & $126,0 \mathrm{a}^{1}$ & $38,6 \mathrm{a}$ & $24,4 \mathrm{ab}$ & $45,4 \mathrm{ab}$ & $17,6 \mathrm{~b}$ \\
FC 70\% + ABO 30\% & $116,4 \mathrm{a}$ & $38,0 \mathrm{a}$ & $27,8 \mathrm{a}$ & $33,4 \mathrm{~b}$ & $26,4 \mathrm{a}$ \\
Tableta FC & $82,6 \mathrm{bc}$ & $36,2 \mathrm{a}$ & $30,4 \mathrm{a}$ & $49,6 \mathrm{a}$ & $31,2 \mathrm{a}$ \\
FC 50\% + FP 50\% & $96,0 \mathrm{~b}$ & $30,2 \mathrm{ab}$ & $25,6 \mathrm{ab}$ & $38,6 \mathrm{ab}$ & $28,0 \mathrm{a}$ \\
FP 70\% + AS 30\% & $67,4 \mathrm{c}$ & $24,6 \mathrm{~b}$ & $18,8 \mathrm{~b}$ & $33,8 \mathrm{~b}$ & $29,4 \mathrm{a}$ \\
\hline
\end{tabular}

${ }^{1}$ Medias con igual letra en la misma columna, no difieren estadísticamente según prueba de separación de rangos LSD Fisher ( $\leq \leq 0,05$ ). / Means with the same letter in the same column do not differ statistically according with Fishers least significant difference test LSD $(\mathrm{p} \leq 0,05)$.

FC 40\% + FP 40\% + ABO 20\%= fibra de coco al 40\% + fibra de palma al 40\% + abono orgánico comercial al 20\% / coconut fiber 40\% + oil palm leaf fiber $40 \%+$ commercial organic compost $20 \%$.

FC 50\% + FP 50\% = fibra de coco al 50\% + fibra de palma al 50\% / coconut fiber 50\% + oil palm leaf fiber 50\%.

FP 70\% + ABO 30\%= fibra de palma al 70\% + abono orgánico comercial al 30\% / oil palm leaf fiber 70\% + commercial organic compost $30 \%$.

FP 70\% + AS 30\% = fibra de palma al 70\% + aserrín al 30\% / oil palm leaf fiber 70\% + sawdust 30\%.

Tableta $\mathrm{FC}=$ tableta comercial de fibra de coco / commercial coco coir slabs. 
no se diferenciaron estadísticamente; estos dos últimos sustratos tampoco se diferenciaron significativamente del tratamiento FP 70\% + AS 30\%, que mostró el menor número de frutos. Considerando la distribución porcentual, esta fue la categoría con menor representación, únicamente entre 10 y $13 \%$ del total de frutos. Para el tamaño de frutos XL tres sustratos sobresalieron: Tableta FC, FC 40\% + FP 40\% + ABO 20\% y FC 50\% + FP 50\%, con una distribución porcentual entre 18 y $21 \%$ del total de frutos. Estos últimos dos tampoco difirieron estadísticamente de los tratamientos con menor número de frutos de esta categoría. Frutos con curvatura acentuada fue en su mayoría la principal causa de deformación encontrada y es lo que ocasiona el rechazo comercial, sin embargo, fue un problema poco frecuente en este experimento. El menor número de frutos rechazados se dio con el sustrato FC 40\% + FP $40 \%$ + ABO 20\% (7\% de los frutos totales), diferenciándose estadísticamente de los demás. Ese último sustrato por lo tanto, se mostró como el de mayor prolificidad en todas las categorías exceptuando la de rechazo, siendo esto altamente conveniente desde el punto de vista comercial.

El Cuadro 6 muestra los parámetros de productividad total registrados en el ciclo completo del cultivo. Siendo congruente con el número de frutos producidos, al valorar los rendimientos totales al final del ciclo del cultivo para cada sustrato evaluado, el tratamiento FC 40\% + FP 40\% + ABO 20\% fue el que obtuvo una producción de 598,63 $\mathrm{kg}$, muy similar a la producción total del sustrato FC 70\% + ABO 30\%. Seguido a los anteriores y sin diferencia estadística está el tratamiento testigo en tabletas de fibra de coco. La tendencia observada para esos tres sustratos fue mantenida en los otros parámetros de rendimiento como número total de frutos por planta, kilos de fruto por planta y kilos por metro cuadrado.

La tableta de fibra de coco es comparable estadísticamente también con la mezcla FC 50\% + FP 50\%, en las variables de productividad total y rendimiento de frutos $\left(\mathrm{kg} /\right.$ planta y $\left.\mathrm{kg} / \mathrm{m}^{2}\right)$. El sustrato FP $70 \%+\mathrm{AS} 30 \%$ obtuvo el menor rendimiento de todos los sustratos evaluados, $44 \%$ inferior cuando se compara con la mezcla de mayor productividad.

Cuadro 6. Productividad total por área, número de frutos por planta y rendimiento $(\mathrm{kg})$ en frutos por planta y por metro cuadrado, en plantas de pepino holandés variedad Fuerte establecidas en diferentes sustratos en condiciones de invernadero. Estación Experimental Agrícola Fabio Baudrit Moreno, Alajuela, Costa Rica. 2013.

Table 6. Total area productivity, number of fruits per plant and fruit yield per plant and per area (square meter) in Dutch cucumber plants, variety Fuerte, grown on different substrates under greenhouse conditions. Fabio Baudrit Moreno Agricultural Experiment Station, Alajuela, Costa Rica. 2013.

\begin{tabular}{lcccc}
\hline & $\begin{array}{c}\text { Productividad total } \\
\text { (kg) en el área } \\
\text { evaluada }\end{array}$ & $\begin{array}{c}\text { Número de frutos } \\
\text { por planta }\end{array}$ & $\begin{array}{c}\text { Rendimiento frutos } \\
\text { por planta }(\mathbf{k g})\end{array}$ & $\begin{array}{c}\text { Rendimiento } \\
\mathbf{k g} / \mathbf{m}^{2}\end{array}$ \\
Tratamiento & $598,63 \mathrm{a}^{1}$ & $12,6 \mathrm{a}$ & $5,99 \mathrm{a}$ & $15,57 \mathrm{a}$ \\
FC 40\% + FP 40\% + ABO 20\% & $594,08 \mathrm{a}$ & $12,1 \mathrm{a}$ & $5,94 \mathrm{a}$ & $15,44 \mathrm{a}$ \\
FC 70\% + ABO 30\% & $567,55 \mathrm{ab}$ & $11,5 \mathrm{a}$ & $5,68 \mathrm{ab}$ & $14,77 \mathrm{ab}$ \\
Tableta FC & $510,66 \mathrm{~b}$ & $10,9 \mathrm{~b}$ & $5,11 \mathrm{~b}$ & $13,29 \mathrm{~b}$ \\
FC 50\% + FP 50\% & $398,7 \mathrm{c}$ & $8,7 \mathrm{c}$ & $3,99 \mathrm{c}$ & $10,37 \mathrm{c}$ \\
FP 70\% + AS 30\% &
\end{tabular}

${ }^{1}$ Medias con igual letra en la misma columna, no difieren estadísticamente según prueba de separación de rangos LSD Fisher (P $\left.\leq 0,05\right)$. / Means with the same letter in the same column do not differ statistically according to Fishers least significant difference test LSD $(\mathrm{p} \leq 0.05)$.

FC $40 \%$ + FP 40\% + ABO 20\%= fibra de coco al 40\% + fibra de palma al 40\% + abono orgánico comercial al 20\% / coconut fiber 40\% + oil palm leaf fiber $40 \%+$ commercial organic compost $20 \%$.

FC 50\% + FP 50\% = fibra de coco al 50\% + fibra de palma al 50\% / coconut fiber 50\% + oil palm leaf fiber 50\%.

FP $70 \%+$ ABO 30\% = fibra de palma al $70 \%$ + abono orgánico comercial al 30\% / oil palm leaf fiber 70\% + commercial organic compost $30 \%$.

FP 70\% + AS 30\%= fibra de palma al 70\% + aserrín al 30\% / oil palm leaf fiber 70\% + sawdust 30\%.

Tableta FC= tableta comercial de fibra de coco / commercial coco coir slabs. 
Sobre la distribución porcentual por categoría comercial del rendimiento (variable peso), se observó en todos los sustratos predominancia del tamaño S y un porcentaje de rechazo que no superó el $10 \%$ en todos los casos (Figura 2). Al unir el peso de los frutos de tamaño L y XL (de mayor valor comercial), no se superó el 50\% de todos los frutos cosechados en estas categorías en ninguno de los sustratos evaluados.

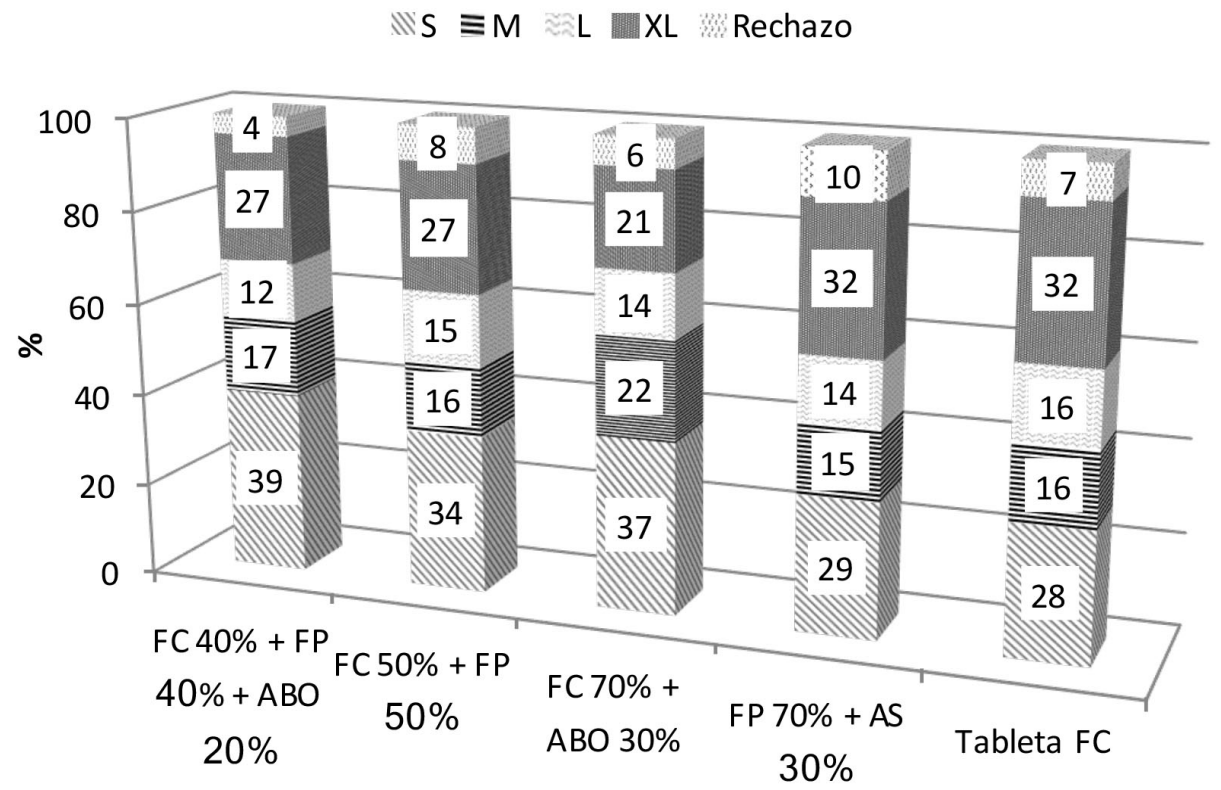

\section{Sustratos}

Figura 2. Distribución porcentual del peso de la producción por categoría comercial, en plantas de pepino holandés variedad Fuerte establecidas en diferentes sustratos en condiciones de invernadero. Estación Experimental Agrícola Fabio Baudrit Moreno, Alajuela, Costa Rica. 2013.

FC 40\% + FP 40\% + ABO 20\%= fibra de coco al 40\% + fibra de palma al 40\% + abono orgánico comercial al 20\%; FC $50 \%+$ FP 50\% = fibra de coco al 50\% + fibra de palma al 50\%; FP 70\% + ABO 30\%= fibra de palma al 70\% + abono orgánico comercial al 30\%; FP 70\% + AS 30\%= fibra de palma al 70\% + aserrín al 30\%; Tableta FC= tableta comercial de fibra de coco.

Figure 2. Percentage distribution of production weight by commercial category in Dutch cucumber plants, variety Fuerte, grown on different substrates under greenhouse conditions. Fabio Baudrit Moreno Agricultural Experiment Station, Alajuela, Costa Rica. 2013.

$\mathrm{FC} 40 \%+\mathrm{FP} 40 \%+\mathrm{ABO} 20 \%=$ coconut fiber $40 \%+$ oil palm leaf fiber $40 \%+$ commercial organic compost $20 \%$; FC 50\% + FP 50\%= coconut fiber 50\% + oil palm leaf fiber 50\%; FP 70\% + ABO 30\%= oil palm leaf fiber 70\% + commercial organic compost 30\%; FP 70\% + AS 30\%= oil palm leaf fiber 70\% + sawdust 30\%; Tableta FC= commercial coco coir slabs.

\section{Discusión}

\section{Retención de humedad}

Los sustratos donde se observó mayor retención de humedad, tienen en común la presencia de la fibra de coco, que tiene entre sus características físicas la capacidad de retener de tres a cuatro veces su propia peso en agua, debido a la estructura de sus finos poros (Abad et al., 2002), tal y como ocurrió con la mezcla de sustrato con 
$70 \%$ de fibra de coco. En el caso de la tableta comercial de fibra de coco, además de la propia fibra, una ventaja adicional de ese tratamiento es que ese material está protegido por el plástico que envuelve la tableta, por lo que, la superficie expuesta del sustrato es mínima, limitándose al orificio donde se coloca la plántula al inicio del ciclo; así, la pérdida de agua en el sustrato por evaporación es muy reducida. Lo contrario sucede cuando se siembra en maceteros donde el área expuesta del sustrato es mayor. Esto es más perjudicial en las primeras semanas del cultivo, cuando el dosel de la planta aún es pequeño y la biomasa de la planta no es suficiente para ofrecer sombreo y reducir la evaporación de la superficie expuesta del sustrato. Ese problema se intensifica más si se presentan altas temperaturas en el invernadero.

El problema de baja retención de humedad de los sustratos con 70 y $50 \%$ de fibra de hoja de palma aceitera en la mezcla, se puede relacionar con la presencia de partículas de tamaño mayor en ese tipo de fibra. Se han indicado alrededor de $82 \%$ de partículas con tamaño superior a $2 \mathrm{~mm}$ en fibra de hojas de palma aceitera, lo que se considera alto (Meneses-Fernández y Quesada-Roldán, 2015). Un comportamiento muy similar al evaluar otra fibra de la palma Archontophoenix alexandrae, para extracción de palmito, lo reportaron también Fermino et al. (2014), quienes encontraron alta porosidad total y espacio aéreo, pero poca retención de agua. Partículas grandes estimulan la presencia de macroporos y la aireación, pero tienen poca retención favoreciendo el drenaje. En esas mezclas de sustratos, la presencia de aserrín de melina y fibra de coco, ambos materiales caracterizados por ofrecer buena retención de humedad, no fue en la cantidad suficiente para garantizar mayor retención de humedad.

\section{Crecimiento}

El aporte nutricional del abono orgánico utilizado debido a la formación y presencia en el medio de sustancias húmicas, y el alto contenido en bases ( $\mathrm{Ca}, \mathrm{Mg}$ y K), aunado a la retención de agua y aireación que le brindaron los otros componentes de la mezcla (fibra de coco y fibra de palma, respectivamente) podrían explicar por qué en los sustratos fibra de coco al $40 \%$ + fibra de palma al $40 \%$ + abono orgánico comercial al 20\%, y fibra de coco al $70 \%$ + abono orgánico comercial al 30\%, se observó mayor crecimiento en las variables de altura de planta y tamaño y número de hojas. Otros estudios han demostrado efectos benéficos de la combinación de sustratos con buen aporte nutricional y materiales que aseguran oxigenación y aporte de agua que garantizan la debida proporción de macro y microporos del sustrato. Lo anterior fue demostrado por Choi et al. (2012) al evaluar diversos sustratos con fórmulas nutritivas de base ya incorporadas en el medio, en almácigos injertados de varios tipos de hortalizas.

Debido a la dificultad de la fibra de palma para retener la humedad, fue notorio como el sustrato compuesto en un $70 \%$ por ese material se secaba más rápido que los otros tratamientos, principalmente en los días que se registraron las temperaturas más altas dentro del invernadero. Como consecuencia hubo rezago en el crecimiento de las plantas cultivadas en este sustrato. Para esa fibra, Meneses-Fernández y Quesada-Roldán (2015) reportaron una baja capacidad de retención de agua, cercana al 31\%, cuando lo ideal es de al menos 50\% (Fonteno, 1996). Ese sustrato, ofrece alta concentración de P y K (Meneses-Fernández y Quesada-Roldán, 2015) y tiene predominantemente en su composición los macroporos (aquellos con diámetro superior a $30 \mu \mathrm{m}$ ) que, permiten el intercambio gaseoso del sustrato y no los microporos que, son los responsables por la retención de la humedad. Respuestas como la anterior ejemplifican como en la selección de un sustrato deben prevalecer criterios físicos por sobre aspectos químicos (Fermino, 2014).

La precocidad en la producción es una característica importante para el productor, dado que disminuye el tiempo para empezar a cosechar y obtener retorno económico con la producción. Cuanto mejor la proporción de partículas grandes y pequeñas del medio, se tiene un sustrato más balanceado que ofrece buena retención de humedad pero a la vez aireación, consecuentemente, se mejora la disponibilidad de nutrientes para la planta y se 
obtiene un crecimiento más equilibrado entre las fases fenológicas (Cruz et al., 2010). Al carecer el tratamiento fibra de palma al 70\% + aserrín al 30\% de una capacidad de retención de agua adecuada, aunado a posibles limitantes nutricionales por la falta de otros componentes de mayor contenido orgánico y menor lignificación en esa mezcla, desfavoreció el proceso de floración en las dos fechas evaluadas (19 y 25 DDT), y por ende, afecto también la productividad.

En los tratamientos con menos agua disponible, los resultados en la floración y fructificación fueron opuestos a lo encontrado por Quesada (2015), quien reportó cómo la falta de humedad en sustratos de fibra de coco aceleró la floración y fructificación como estrategia de reproducción y sobrevivencia ante el estrés hídrico en plantas de chile dulce. Se desprende por tanto, de estos resultados, que la planta de pepino es muy sensible a condiciones de poca humedad en el medio, reflejado en el menor crecimiento vegetativo y la capacidad reproductiva de la planta.

\section{Producción}

La predominancia de frutos tamaño $\mathrm{S}$ en todos los sustratos, pudo deberse a aspectos de adaptación del genotipo en su relación con las condiciones del ambiente y no al sustrato, puesto que, aún en los sustratos que ofrecieron mejores condiciones de desarrollo para la planta, predominó la categoría de tamaño menor de la fruta. Pocos frutos fueron categorizados como rechazo en cualquiera de los sustratos evaluados. La deformación en esos casos se asocia, principalmente en materiales partenocárpicos como el utilizado, a problemas por polinización cruzada causada por la presencia de agentes polinizadores que ingresan polen de otras fuentes (Hochmuth, 2012). El invernadero en el que se trabajó no estaba sembrado exclusivamente con plantas de pepino, pues en el mismo sitio se tenían plantadas otras especies de la familia Cucurbitaceae, caso del melón y el ayote, siendo factible que hubiese ocurrido algún pequeño porcentaje de cruzamiento y contaminación de polen.

Los valores de rendimiento obtenidos en los mejores tratamientos de esta investigación, fueron similares a la producción de pepino que se reporta en la literatura, entorno de 15 y $16 \mathrm{~kg} / \mathrm{m}^{2}$ (López-Elías et al., 2011; Reche, 2011). Se informó de una productividad de alrededor de 5 a $7 \mathrm{~kg}$ por planta, semejante a la obtenida (Quesada et al., 2008), sin embargo, los valores de rendimiento alcanzados fueron inferiores a los informados por ChacónPadilla y Monge-Pérez (2016), quienes reportaron una producción entre 6,9 y 9,9 kg/planta y entre 18 y $25 \mathrm{~kg} / \mathrm{m}^{2}$. Factores como precocidad de la cosecha, genotipo escogido, tipos de podas, condiciones de clima (temperatura principalmente) y aspectos fitosanitarios y nutricionales, inciden directamente en la variación de la productividad del pepino en invernadero, que del mismo modo es muy superior a la que puede ser obtenida cultivando al aire libre (Hochmuth, 2012).

En el caso de las mezclas de sustrato fibra de palma + fibra de coco + abono orgánico y fibra de coco + abono orgánico, el buen balance físico y químico de los aportes de cada uno de los componentes se reflejó en el resultado obtenido en productividad. Con las fibras se brinda volumen y porosidad al medio (macroporos con la fibra de palma y microporos con la fibra de coco), mientras que el compost ayuda también con la retención de humedad y brinda además un aporte nutricional orgánico que favorece el desarrollo de la planta y la hace menos dependiente a la solución nutritiva (Acosta, 2012). Las referidas mezclas de sustratos resultaron tan productivas como el testigo comercial, lo que demuestra la opción alternativa que estos sustratos pueden representar en la producción comercial de pepino holandés.

La mezcla en la que se usó la mayor proporción de fibra de hoja de palma (70\%) tuvo el peor comportamiento entre los sustratos, en todos los parámetros de rendimiento total, lo cual se relacionó con la dificultad de retención de agua de este material. Además del aserrín, el otro componente de la mezcla, pese a tener una retención de humedad cercana al 57\% (Quesada y Méndez, 2005), no fue lo suficiente para compensar y llenar el espacio poroso vacío presente por la fibra de palma. El bajo contenido de humedad de esos sustratos afectó el rendimiento del cultivo, 
tal y como Quesada (2015) encontró en chile dulce. En esa ocasión se observó una reducción de aproximadamente $18 \%$ en el rendimiento comercial cuando las plantas se sometían a un estrés hídrico, consecuentemente, disminuyó el agua y nutrimentos disponibles en sustratos de fibra de coco. El limitado desarrollo vegetativo de las plantas en el sustrato de fibra de palma al 70\% + aserrín al 30\%, especialmente en las primeras etapas de crecimiento, influyó en la producción de fotoasimilados producidos y se reflejó posteriormente en la producción alcanzada. La mezcla de palma y fibra de coco (50\% de cada componente) tuvo el segundo peor rendimiento, aunque estadísticamente no difirió de la tableta de fibra de coco en las variables de productividad total y rendimiento en kilogramos por planta y por metro cuadrado. De estos resultados, se desprende la importancia de validar las mezclas propuestas al momento de conformar un sustrato, pues la fibra de palma al 70\% o al 50\%, no dio la respuesta adecuada en productividad que sí se observó en esa misma fibra de palma al $40 \%$ en combinación con los otros materiales componentes del sustrato.

Los frutos de tamaño $\mathrm{S}$ predominaron en la producción. Si bien esos frutos son exportables, es poco deseable que prevalezca ese tamaño de fruta. En producciones comerciales deben prevalecer frutos tamaño L y XL, que son los de mayor importancia por su retorno económico en la mayoría de los mercados donde este tipo de pepino se comercializa (Mora et al., 2007). Además, la distribución por tamaño atiende criterios de exportación para el mercado norteamericano, y lo esperado en condiciones normales son frutos entre 32 y $38 \mathrm{~cm}$ (Mora et al., 2007), lo que abarcaría en esta clasificación las categorías M, L y XL. Mientras que el porcentaje de peso de frutos tamaños S o M varió entre 44 y 59\% (el sustrato $\mathrm{FC} 70 \%$ + ABO 30\% fue el que registró más peso de frutos en esas dos categorías), únicamente se obtuvo entre 35 y $48 \%$ de frutos sumando las categorías L y XL. En ese último caso, fue en la Tableta FC que se cosechó el mayor porcentaje de frutos de esos tamaños. Probablemente la interacción genotipo-ambiente fue el principal motivo para este comportamiento (Chacón-Padilla y Monge-Pérez, 2016). Las propiedades fisicoquímicas de los sustratos modifican la capacidad de absorción de $\mathrm{N}$ de la planta, siendo que ese elemento es determinante en la relación fuente-sumidero de la planta de pepino y consecuentemente, en la respuesta en la formación de los frutos y la productividad total (Dai et al., 2011). Temperaturas altas (superiores a $34{ }^{\circ} \mathrm{C}$ ), conforme lo registrado algunos días, pudieron contribuir también a esa respuesta que perjudicó la formación de frutos de mayor tamaño, pues Guo et al. (2008), reportaron también influencia directa de la temperatura en la producción, incluso por sobre factores como la fertilización nitrogenada.

Finalmente, debe destacarse el bajo porcentaje de frutos en la categoría de rechazo, $10 \%$ o menos en todos los sustratos evaluados, indicador de buen manejo del cultivo. En ese particular resultó notorio nuevamente el sustrato FC $40 \%$ + FP 40\% + ABO 20\%, ya identificado entre los de mayor productividad y solo con $4 \%$ del peso de los frutos totales con la inconveniencia de rechazo.

\section{Conclusiones}

El crecimiento y la productividad de plantas de pepino holandés variedad Fuerte, fue de similar comportamiento en sustrato con fibra de coco al $40 \%$ + fibra de palma al $40 \%$ + abono orgánico comercial al 20\%, y fibra de coco al $70 \%$ + abono orgánico al 30\%, en relación con el sustrato comercial de fibra de coco empacada en tabletas, atribuido al aporte nutricional del abono orgánico junto con la aireación y retención de humedad de las fibras. La fibra de coco y el abono orgánico confirmaron su valía como sustratos alternativos. Por su parte, sustratos con alta proporción de fibra de palma en su composición, presentaron problemas para retener humedad en el sustrato y consecuentemente, se reflejó bajo desarrollo de la planta y un rendimiento limitado.

La proporción correcta en la que deben combinarse los materiales constituye el factor clave en la respuesta de la planta, pues los resultados reflejaron un comportamiento positivo y otro negativo para un mismo elemento componente de las mezclas, como fue el caso de la fibra de hoja de palma, siendo que ese material debe ser cuidadosamente combinado para obtener un buen resultado. 


\section{Literatura citada}

Abad, M., P. Noguera, R. Puchades, A. Maquieira, and V. Noguera. 2002. Physico-chemical and chemical properties of some coconut coir dusts for use as a peat substitute for containerized ornamental plants. Bioresour. Technol. 82:241-245. doi:10.1016/S0960-8524(01)00189-4

Acosta, C.M. 2012. Selección de sustratos para horticultura. Redes Editores, Morelos, MEX.

Alvarado, M.A., y J.A. Solano. 2002. Producción de sustratos para viveros. VIFINEX-OIRSA, CRC.

Chacón-Padilla, K., y J.E. Monge-Pérez. 2016. Evaluación del rendimiento y la calidad de seis genotipos de pepino (Cucumis sativus L.) cultivados bajo invernadero en Costa Rica. Rev. Colomb. Cienc. Hort. 10:323-332. doi:10.17584/ rech.2016v10i2.5069

Choi, J.M., C.W. Lee, and J.P. Chun. 2012. Optimization of substrate formulation and mineral nutrition during the production of vegetable seedling grafts. Hortic. Environ. Biotechnol. 53:212-221. doi:10.1007/s13580-012-0108-1

Cruz, E., M. Sandoval, V. Volke, V. Ordaz, J.L. Tirado, y J. Sánchez. 2010. Generación de mezclas de sustratos mediante un programa de optimización utilizando variables físicas y químicas. Terra Latinoam. 28:219-229.

Dai, J., S. Liu, W. Zhang, R. Xu, W. Luoa, S. Zhang, X. Yin, L. Han, and W. Chen. 2011. Quantifying the effects of nitrogen on fruit growth and yield of cucumber crop in greenhouses. Sci. Hort. 130:551-561. doi:10.1016/j.scienta.2011.08.004

Fermino, M.H. 2014. Substratos: composição, caracterização e métodos de análise. Agrolivros, Rio Grande do Sul, BRA.

Fermino, M.H., R.S. Gonçalves, J.R.P. Silveira, A. Battistin, M. Trevisan, e A.C. Busnello. 2014. Fibra de palmeira como substrato para hortaliças. Hortic. Bras. 32:404-408. doi:10.1590/S0102-053620140000400006

Fonteno, W. 1996. Sustratos: tipos y propiedades físicas y químicas. In: D. Reed, editor, Guía del productor: Agua, sustratos y nutrición en los cultivos de flores bajo invernadero. Ball publishing, HortiTecnia Ltda, COL. p. 93-123.

Guo, R., X. Li, P. Christie, Q. Chen, and F. Zhang. 2008. Seasonal temperatures have more influence than nitrogen fertilizer rates on cucumber yield and nitrogen uptake in a double cropping system. Environ. Pollut. 151:443-451. doi:10.1016/j. envpol.2007.04.008

Handreck, K., and N. Black. 2002. Growing media for ornamental plants and turf. $3^{\text {th }}$ ed. UNSW Press, AUS.

Hernández-Apaolaza, L., A.M. Gascó, J.M. Gascó, and F. Guerrero. 2005. Reuse of waste materials as growing media for ornamental plants. Bioresour. Technol. 96:125-131. doi:10.1016/j.biortech.2004.02.028

Hernández-González, Z., J. Sahagún-Castellanos, P. Espinosa-Robles, M.T. Colinas-León, y J.E. Rodríguez-Pérez. 2014. Efecto del patrón en el rendimiento y tamaño de fruto en pepino injertado. Rev. Fitotec. Mex. 37:41-47.

Hochmuth, R.C. 2012. Greenhouse cucumber production-Florida greenhouse vegetable production handbook. Vol 3. IFAS Extension, University of Florida, FL, USA. http://edis.ifas.ufl.edu/pdffiles/CV/CV26800.pdf (accessed 27 feb. 2015).

Larcher, W. 2000. Ecofisiología vegetal. RiMa Editora, São Carlos, BRA.

López-Elías, J., J.C. Rodríguez, M.A. Huez, S. Garza, J. Jiménez, y E.I. Leyva. 2011. Producción y calidad de pepino (Cucumis sativus L.) bajo condiciones de invernadero usando dos sistemas de poda. Idesia 29(2):21-27. doi:10.4067/S071834292011000200003

Méndez, C. 2007. Sustratos para la producción intensiva de hortalizas en Costa Rica. Rev. Gestión Hortíc. 2(10):9-13.

Meneses-Fernández, C., y G. Quesada-Roldán. 2015. Análisis de sustratos orgánicos alternativos para la producción de hortalizas en invernadero en Costa Rica. Invest. Agropecu. 12:9-16. 
Mora, N., H. Campos, J. Fallas, S. Morales, M. Blanco, y J.A. Alfaro. 2007. Caracterización de la agrocadena pepino holándes. Ministerio de Agricultura y Ganadería, CRC.

Nelson, P. 2003. Greenhouse operation and management. $6^{\text {th }}$ ed. Prentice Hall, USA.

Quesada, G. 2015. Producción de chile dulce en invernadero bajo diferentes niveles de agotamiento en la humedad del sustrato. Agron. Costarricense 39:25-36.

Quesada, G., y C. Méndez. 2005. Análisis fisicoquímico de materias primas y sustratos de uso potencial en almácigos de hortalizas. Rev. Agric. Trop. 35:1-13.

Quesada, G., N. Umaña, y F. Marín. 2008. La selección del sustrato y el rendimiento comercial de plantas de pepino holandés. Bol. ProNap. 2(10):4-6.

Quintero, M.F., C.A. González, y J.M. Guzmán. 2011. Sustratos para cultivos hortícolas y flores de corte. En: V.J. Florez, editor, Sustratos, manejo del clima, automatización y control en sistemas de cultivo sin suelo. Editorial Universidad Nacional de Colombia, COL. p. 79-108.

Quirós, J.I. 2015. Mercado mundial de pepino. Bol. ProNap 9(53):9-13.

Reche, J. 2011. Cultivo del pepino en invernadero. Ministerio de Medio Ambiente y Medio Rural y Marino, Madrid, ESP.

Shaw, N.L., D.J. Cantliffe, and P.J. Stofella. 2007. A new crop for North American greenhouse growers: Beit Alpha cucumber - progress of production technology through university research trials. Act. Hort. 731:251-258. doi:10.17660/ ActaHortic.2007.731.34

Takane, R., S. Yanagisawa, e E. Góis, 2013. Técnicas em substratos para a floricultura. Expressão Gráfica e Editora, Fortaleza, BRA. 